See discussions, stats, and author profiles for this publication at: https://www.researchgate.net/publication/305523887

\title{
Modelling the particles impingement angle to produce maximum erosion
}

Article in Powder Technology · November 2016

DOI: 10.1016/j.powtec.2016.07.041

CITATIONS

25

3 authors:

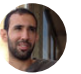

Yaron Ben Ami

Technion - Israel Institute of Technology

11 PUBLICATIONS 77 CITATIONS

SEE PROFILE

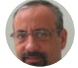

Avi Levy

Ben-Gurion University of the Negev

197 PUBLICATIONS 3,286 CITATIONS

SEE PROFILE

Some of the authors of this publication are also working on these related projects:

Project Energy absorption in particle-wall impact View project

Project Study of shock reflection in overexpanded supersonic jets View project
READS

867

Avi Uzi

Ben-Gurion University of the Negev

19 PUBLICATIONS 118 CITATIONS

SEE PROFILE 


\title{
Modelling the Particles Impingement Angle to Produce Maximum Erosion
}

\author{
Y. Ben Ami*, A. Uzi, A. Levy \\ Department of Mechanical Engineering, Ben-Gurion University of the Negev, PO Box 653, Beer-Sheva 81405, Israel. \\ * Corresponding author. Fax: +972 86472813; Email address: yaronhab @ post.bgu.ac.il (Y. Ben Ami).
}

\begin{abstract}
A semi-mechanistic, semi-empirical erosion model was developed and validated by experimental data. The novelty of the developed model is by accurately predicting the impingement angle that produce maximum erosion and relating it to measurable mechanical properties such as hardness and fracture toughness. The ratio of the target material fracture toughness $(R)$ to its hardness $(H)$ is the parameter which governs the erosion mechanism and consequently the angle for maximum erosion. It was noticed that for material with high $R / H$, i.e. ductile materials, the preferred mode is ploughing, a process analogous to rubbing in which the penetrating particle squeeze the target material out of the indentation to preform ridges, which are knocked off at subsequent impacts. The lower the ratio $R / H$ is, a tendency to fracture mechanism occurs. The fracture mechanism is a process very similar to brittle chipping during metal cutting, where the applied force propagates cracks parallel to the surface. A simplified model, defining the horizontal force which is applied on the particle in its movement through the material, was introduced. This simplified model takes into account the relative effect of each of the ploughing and fracture mechanism on the total material removal. The material parameter which governs the relative effect of the mechanisms is $R / H$. Particle angularity also contributes to decreasing of the fracture mechanism, hence a governing non-dimensional number was received, $R / H \cdot d_{\text {eff }}$, where $d_{\text {eff }}$ is the effective diameter of the penetrating particle tip. Incorporating this parameter in the erosion model yielded good agreement with experimental data and was able to explain the phenomenon of different maximum erosion angle and velocity exponent for different target materials. The relation between the developed model empirical coefficients to target material mechanical properties was studied for eight different materials, ranging from soft metals to hardened stainless steel. Correlations between model coefficients to $R / H$ were developed and exhibited good agreement with mechanistic analysis.
\end{abstract}

Keywords: Erosive wear; Erosion modelling; Solid particle impact; Impingement angle;

\section{Introduction}

Erosive wear of pipe components during conveying of solid particles is a major industrial problem. Conveying material through pipe systems is a common industrial process in the mining industry, chemical industry, pharmaceutical industry, food processing, energy producing, oil refining and so on. The erosion caused during conveying shortens the lifetime of the system components which yields substantial maintenance costs and can even bring a system to failure. Evaluation of the 
Nomenclature

English Letters

A

$b$

$C_{C}, C_{D}$

$D$

$d$

E

e

F

$f$

H

$K_{c}$

$L$

$m$

$\Delta Q$

$R$

$U$

w

$y$

\section{Greek Letters}

$\alpha$

$\beta$

$\Delta \varepsilon$

$\varepsilon_{f}$

$\eta$

$\rho$

$\tau$

\section{Subscripts}

C

D

eff

$\max$

$p$

$x$

$y$

0 surface area

fatigue ductility exponent

model empirical coefficients

indentation diameter

particle diameter

target material Young modulus

restitution coefficient

force

model empirical exponent

target material hardness

critical stress intensity factor

indentation length

particle mass

indentation volume

target material fracture toughness

particle impact velocity

indentation width

indentation depth

particle impingement angle

particle rake angle

Strain amplitude

fatigue ductility coefficient

particle shape factor

density

shear stress

$\mathrm{kg} / \mathrm{m}^{3}$

$\mathrm{Pa}$ rad

rad

$m^{2}$

$m$

$m$

$\mathrm{Pa}$

$N$

$P a \cdot m^{1 / 2}$

$\mathrm{Pa}$

$m$

$k g$

$J / m^{2}$

$m / s$

$m$

$m$

rad

related to 'cutting' mechanism

related to 'deformation' mechanism

effective value

maximum value

related to the particle

in $x$ direction

in $y$ direction

initial value 
erosion rate of the pipe system components, in terms of the flow conditions, is very beneficial for the prediction of the lifetime expectancy of the component and helps design a better erosion resistant system.

Erosion by solid particles conveying is a complex problem which incorporates several fields of research: (i) the erosive wear is caused by impacts of solid particles with the pipe wall. The mechanics of the particle-wall impact and the wall material removal at impact encompass the field of contact mechanics and fracture mechanics. (ii) On the other hand, the boundary conditions for this impact, i.e. the impact velocity and angle, are controlled by the multiphase flow of the carrier fluid and the particles themselves. Hence, erosion rate predictions must include these two aspects. Nowadays, with the large ascent in computational power, the task of calculating particle trajectories and their wall impact conditions in dependence with the multiphase flow conditions is feasible using CFD-DEM numerical simulations. In the last decade numerous efforts (Many of them were reviewed by Njobuenwu \& Fairweather [1] and Parsi et al. [2]) have been made to obtain the wear patterns of the pipe components by implementing a single particle impact erosion model on the simulated impacts data. These efforts focus on the influence of the flow on the erosion. Although these numerical works use the single particle impact erosion models, efforts to improve the accuracy and applicability of these models for a wide range of materials have diminished in the last years.

Efforts of developing analytical erosion models which predict the volume of material removal at single particle impact goes more than 50 years back and many erosion equations were developed under different assumptions and methodologies. The first to explore this direction was Finnie [3]. In his pioneering work, Finnie [3] used a method common in the metal cutting field and some simplifying assumptions in order to calculate the volume removal of a single sharp particle acting as the knife tip of a machining tool. Finnie [3] concluded that the only material property which affects erosion rate is hardness. Although Finnie [3] presented a novel approach, his model suffered from several problems. One major issue with this model is that it does not predict erosion in normal impingement angle. In order to deal with this problem, Bitter [4],[5] introduced the concepts of two erosion mechanisms- 'cutting' and 'deformation'. 'Cutting' is a mechanism much similar to the one described by Finnie [3], and is dominant at low impingement angles. 'Deformation' mechanism was described by Bitter [4],[5] as the work of a repeated plastic deformation caused by the normal component of the particle velocity, which in turn cause crack propagation and essentially material removal. Bitter [4],[5] was not able to assign the erosion rate to known mechanical properties, thus he used two empirical coefficients, one for each mechanism. Hutchings [6] developed a model for the 'deformation' mechanism and suggested that the mechanism is of low-cycle fatigue. Many of the later erosion models, such as Neilson \& Gilchrist [7], Brach [8], Huang et al. [9], Arabnejad et al. [10], adopted this concept of two erosion mechanisms. Nevertheless, the ability to capture the impact angle for maximum erosion in these models was poor and additionally the relation between these angle and measurable mechanical properties remained unclear.

A major problem in erosion modelling is assigning the erosion rate to measurable mechanical properties of the materials, due to the fact that mechanical properties of material are measured on 
large scale test models, while the material removal caused by particle impact is in a much smaller scale. For this reason, almost all of the erosion models incorporate empirical coefficients which have to be calibrated in direct erosion experiments. The common approach in most of the models was that these empirical coefficients are related primarily to the hardness of the target material, since it is a measure of the material resistance to plastic deformation. Finnie's [3] model claims that erosion resistance (inverse to the erosion rate) is linear with the target material hardness, however, experiments [10],[11] showed non-linear relation. Nevertheless, Levy [12] demonstrated that harder material can be less erosion resistant and suggested that the ductility of the material is important in the manner that the material can suffer larger deformation without material removal. Furthermore, Hutchings [13] and Hornbogen [14] claimed that fracture toughness is a dominant factor which effects the erosion mechanism.

The complexity of the influence of material properties on the erosion process and the necessity to have means to predict erosive wear in real industrial systems led some researchers like Oka et al. [15],[16] and Ahlert [17] to develop completely empirical models that fit well with experimental data for specific materials. Oka et al. [15],[16] extended this approach to find a correlation between the empirical constants to target material hardness. While this approach is very practical, academically, these kind of models do not give any insight into the nature of the erosion process and do not give the empirical coefficients any physical interpretation. Here arises the necessity for a semi-mechanistic, semi-empirical model. This kind of model, on the one hand, will capture the nature of the erosion process and the governing mechanical properties of the materials. On the other hand, the model will include coefficients which are calibrated through direct erosion experiments and can be related empirically to measurable mechanical properties. Two models of this kind are Arabnejad et al. [10] and Huang et al. [9]. Arabnejad et al. [10], which used a combination of Finnie's [3] 'cutting' model and Bitter's [4],[5] 'deformation' model together with empirical coefficients and find the relation of these coefficients to material hardness. Yet, the known effect of ductility was not taken into account in this model. Huang et al. [9] divided the erosion mechanisms to tangential and normal velocity effect (analogous to 'cutting' and 'deformation) and made an analysis of the size of indentation produced by a particle impact. For the normal velocity erosion Huang et al. [9] used Hutchings [6] model for the erosion by normal impact angles. Huang et al. [17] combined mechanistic analysis with empirical coefficients, related to hardness and ductility, which should be calibrated in experiments. One key problem with this model is its incapability of predicting a change in the maximum erosion angle for different types of target materials, phenomenon that was well observed in erosion experiments [3],[7],[15],[16].

In the present study, based on the models of Huang et al. [9] and Hutchings [6], a new predictive model for erosion rate in single particle impact is developed. The developed model incorporates the mechanical properties of hardness and fracture toughness and also the model empirical coefficients are associated with these properties. The main superiority of the suggested model is its more solid physical ground, as well as better agreement in predicting the maximum erosion angle for different target materials. 


\section{Theoretical Modeling}

Material removal by particle impact is attributed by two mechanisms, as mentioned. At lower impingement angles, the dominant mechanism is generally called 'cutting'. At that mechanism the vertical component of the particle velocity is consumed to penetrate the target material, while the horizontal component push the particle through the material in a manner that can be described as micromachining, where a chip is cut, or ploughing, where the material is squeezed out of the indentation and piled up in a ridge (The conventionally name of the mechanism 'cutting' is used at this paper, nevertheless the dominant mechanism is actually ploughing, as is discussed later on). The second mechanism 'deformation' is the material removal caused by repeated plastic deformation leads to fatigue crack propagation and essentially to detachment of fragments. The total material removal is the sum of the two mechanisms:

$$
\Delta Q=\Delta Q_{D}+\Delta Q_{C}
$$

Where, $\Delta Q$ is the volume of material removed, $D$ indicates 'deformation' and $C$ indicates 'cutting'.

While 'cutting' mechanism is traditionally referred to as ductile mode and incorporates only friction and plastic deformation analysis, 'deformation' mechanism is analyzed from fracture mechanics point of view. In this theoretical modelling we will show that fracture mechanics considerations are essential not only for deformation mechanism but for 'cutting' mechanism as well.

\section{1. 'Cutting' mechanism modelling}

The volume swept by the particle in its movement through the target material is proportional to the product of the width $w$, maximum depth $y_{\max }$, and the length $L$ of the indentation, as illustrated in Fig.1:

$$
\Delta Q_{C}=c_{1} \cdot w \cdot L \cdot y_{\max }
$$

Where $c_{l}$ is the ratio of material removal to the deformed volume. This parameter is a measure of the material ability to sustain plastic deformation and thus should be inversely proportional to the material ductility.

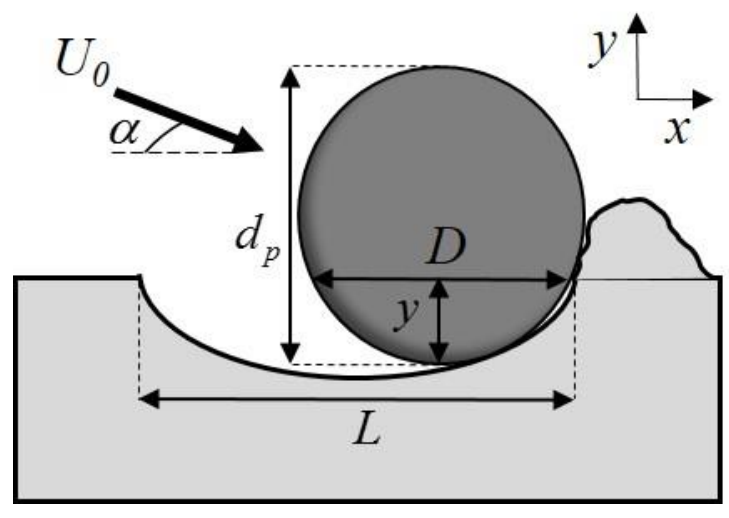

Fig. 1. Illustration of indentation created by particle impact 
The force in the $y$ direction acting on the particle during the impact, assuming constant flow stress, which is proportional to the indentation hardness $H$ :

$$
F_{y}=-A_{y} \cdot H=-\frac{\pi D^{2}}{4} H
$$

where $D$ is the diameter of the indentation. Worth noting that erosion process takes place at the outer layer of the material specimen. Hence the value of the hardness $H$ should be taken as the surface hardness in cases where it differs from the bulk hardness such as for materials that undergo heat treatment or protective coating.

During erosion processes the depth of the indentation is much smaller than the particle diameter $y<<d_{p}$ hence we receive:

$$
\frac{D^{2}}{4}=y\left(d_{p}-y\right) \approx d_{p} \cdot y
$$

Assigning (4) to (3):

$$
F_{y}=-\pi d_{p} H \cdot y
$$

The use of the spherical particle was very convenient for analysis purposes, but it is obvious that real particles are not spherical. Therefore we have to use an effective particle diameter, which is a function of the particle shape, as suggested by Deasle et al. [18]. The sharper the particle is, the smaller the effective diameter is, as protruded in Fig. 2.

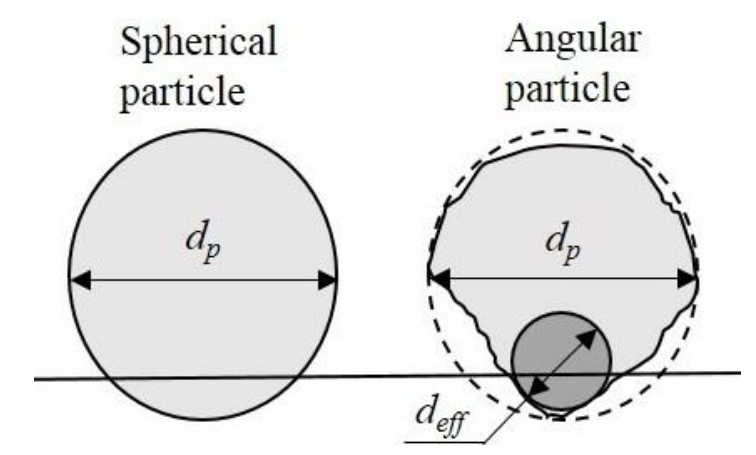

Fig. 2. Illustration of effective diameter of spherical and angular particles

Expressing the force by the means of the effective diameter gives:

$$
F_{y}=-\pi d_{e f f} H \cdot y
$$

Assigning the force into the momentum equation of the particle: 


$$
m_{p} \frac{d U_{y}}{d t}=F_{y}=-\pi d_{e f f} H \cdot y
$$

Applying variable change:

$$
U_{y}=d y / d t
$$

where $U_{y}$ is the particle velocity in $y$ direction. Assigning to Eq. (7):

$$
U_{y} d U_{y}=\frac{-\pi d_{e f f} H}{m_{p}} y d y
$$

Integrating Eq. (9), using the boundary condition: $U_{y}=U_{y 0}$ for $y=0$, where $U_{y 0}$ is the particle initial impact velocity:

$$
\frac{1}{2} U_{y}{ }^{2}=\frac{1}{2} U_{y 0}{ }^{2}-\frac{\pi d_{e f f}}{2 m_{p}} H \cdot y^{2}
$$

The maximum depth of the indentation is reached when the velocity in the $y$ direction is zero:

$$
y_{\max }=\left(\frac{m_{p}}{\pi \cdot d_{\text {eff }} \cdot H}\right)^{\frac{1}{2}} U_{y 0}
$$

In order to calculate the length of the indentation $L$, Huang et al. [9] assumed that the kinetic energy of the horizontal component is proportional to the work of the resistive horizontal force exerted by the target material over a distance $L$ :

$$
\int_{0}^{L} F_{x} d x=\frac{1}{2} m_{p} U_{x 0}^{2}\left(1-e_{x}^{2}\right)
$$

where the restitution coefficient, $e_{x}=U_{x 0} / U_{x f}$ is the ratio of the particle horizontal velocity before and after the impact. In order to evaluate the resistive horizontal force $F_{x}$, Huang et al. [9] assumed a cutting mechanism and suggested that the sharper the particle is, the smaller the resistive area is and made the following assumption:

$$
F_{x} \propto \sigma_{t} A_{x}^{n}
$$

where $\sigma_{t}$ is the tangential stress and $n(0.5 \div 1)$ is a coefficient depending on the particle shape. The sharper the particle, the smaller the resistive area is and hence $n$ is smaller. This assumption does not take into account different mechanisms of material removal and leading to unreasonable result, as presented in Fig. 3, where the Huang et al. [9] 'cutting' model is plotted for various $n$ exponents. 


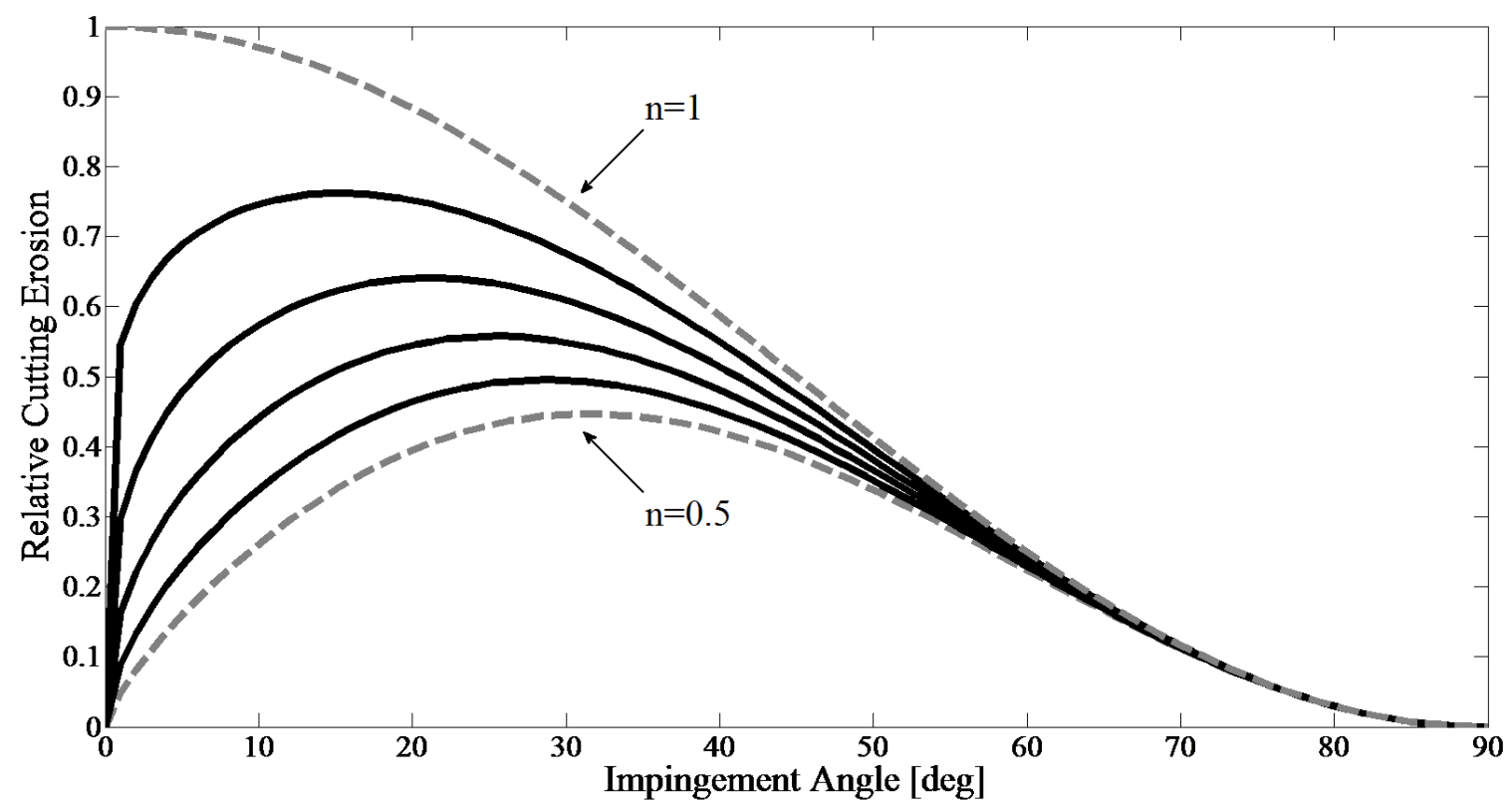

Fig. 3. Huang et al. [9] cutting model as a function of the impingement angle for different $n$ exponents.

As can be seen in Fig. 3 the maximum erosion angle received for spherical particles (large $n$ ) is smaller than for sharp particles, i.e. spherical particles cutting is more efficient. This result is not physical and does not agree with experimental findings [19]. Hence, it can be determined that the mechanism of cutting is not the same for all type of particles and target materials, and should be divided into two cases: ploughing governed mechanism and fracture governed mechanism, as was proposed by Hornbogen [14] and is illustrated in Fig. 4.
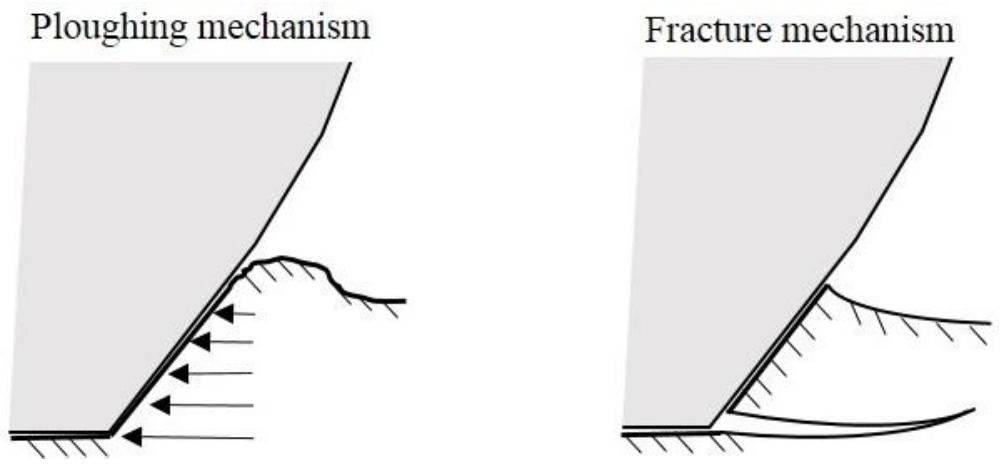

Fig. 4. Illustration of ploughing and fracture mechanisms 


\section{Ploughing mechanism}

Levy [12] examined the nature of particle indentations and did not find evidences to cutting or micromachining mechanisms of material removal. Hutchings [20] studied the erosion mechanism of angular particles and found that the dominant mechanism is ploughing rather than cutting, i.e. the material was pushed into ridges and was not cut as a chip. Analysis of Atkins [21] on transition between cutting and rubbing (analogous to ploughing) in metal cutting process suggests that the transition between the mechanisms depends upon the angle of attack $\beta$, which relates to the particle shape and the non-dimensional parameter $Z=R / \tau \cdot y$, where $R\left[\mathrm{~kJ} / \mathrm{m}^{2}\right]$ is the material fracture toughness, $\tau$ is the shear strength and $y$ is the depth of the cut. During erosion processes the depth of the cut is a derived value, rather than known value as it is in machining. Therefore, for a given penetration depth, the particle effective diameter is the length scale which governs the extent of deformation during the horizontal movement through the target material. Order of magnitude analysis for the ratio of $R / \tau \cdot d_{\text {eff }}$ yields characteristic value of 10 , for $\tau \sim 100 \mathrm{MPa}, R \sim 10 \mathrm{~kJ} / \mathrm{m}^{2}$, typical values of metals and steels and $d_{\text {eff }} \sim 10 \mu \mathrm{m}$, typical length scale of abrasive particle. According to Atkins [28] in this range of $Z$ the transition from ploughing to cutting occurs at attack angle $\beta \approx 70^{\circ}$ (rake angle of $-20^{\circ}$ ), which is about the critical rake angle Hutchings [20] found for micromachining mechanism to occur. This critical angle of attack is very high and due to the rareness of this angle (for rectangular particles with random orientation, only 2 out of 9 particles will have such attack angle), the ploughing mechanism is generally preferred.

For ploughing mechanism, due to its similarity do indentation process, it can be assumed that the horizontal force is proportional to the resistive area, multiplied by constant flow stress:

$$
F_{x} \propto w \cdot y \cdot H
$$

\section{Fracture mechanism}

Fracture occurs during plastic deformation when the energy to separate a crack in the material is smaller than the energy needed to plastically deform the material. The transition between plastic deformation and fracture depends upon material properties and the scale of deformation as well. It is a product of the 'scale effect', which claims that the fracture energy depends upon the area of the crack, while the energy dissipated in plastic deformation process depends upon the deformed volume. In the metal cutting field, this phenomenon leads to brittle chipping- scallops like cuts caused by cracks propagating parallel to the surface in a process comparable to flint-knapping [22]. The same behavior can be assumed during erosion process: When the energy for plastic deformation is too large relative to fracture energy, such in the cases of brittle target material or large scale of deformation caused by large effective diameter, the mechanism transforms into fracture governed mechanism. The process of short parallel cracks propagation is a complex problem with no closed algebraic solution, but on dimensional ground it can be expected that the energy required for separation of a crack will be proportional to the area of the crack:

$$
d E \propto R \cdot w \cdot d L
$$


where $d E$ is the energy required for separation of crack with length $d L, w$ is the width of the crack and $R$ is the fracture toughness, i.e. the energy required for separation of a crack of dimensions $w \cdot d L$.

The source of the energy for the crack growth is the work of the horizontal force exerted by the particle:

$$
d E=F_{x} \cdot d L
$$

Combining (15) and (16) yields:

$$
F_{x} \propto R \cdot w
$$

where $F_{x}$ is the critical load to produce the crack.

For completely 'brittle' mode, it can be seen that the horizontal force does not affected by the depth of penetration. This is due to the fact that the work is consumed to separate the crack and the power required for separation does not depend upon the depth of the cut, but on its surface area.

For a real case, the operating mechanism is a mixed mode of ploughing and fracture. Hence, a model for the combination of the two is required. Atkins [23] proposed a model for metal cutting, which is based on the dissipated energy required to remove a chip of a given volume. Atkins [23] model reads:

$$
\frac{F_{x}}{w}=\tau_{y} t_{0} f_{1}(\alpha, \mu, \phi)+R f_{2}(\alpha, \mu, \phi)
$$

where, $\tau_{y}$ is the shear stress, $t_{0}$ the uncut chip thickness, $R$ the fracture toughness, $\alpha$ the tool rake angle, $\mu$ the friction angle and $\phi$ the shear plane angle.

This model suggest that the two mechanisms contributes separately to the overall required force and there is no mutual effect. In order to model the effect of governing mechanism, such as for ductile material, the energy to extend a crack will be much larger than the energy required for plastic deformation, and the opposite for brittle material, we introduce the following simplified model:

$$
\frac{F_{x}}{w}=\left(a_{1} \cdot H \cdot y\right)^{f}\left(a_{2} \cdot R\right)^{1-f}
$$

where $a_{1}, a_{2}$ are constants and $f$ is an exponent lies between 0 and 1 which is governed by the relative part of the ploughing and fracture mechanisms. The larger the ploughing mechanism dominance is, the plastic work significance is larger and hence the larger $f$ is. Fig. 5 illustrates the suggested model for resistive horizontal force as a function of the depth of penetration for given $R$, $H$ and different $f$ exponent. 


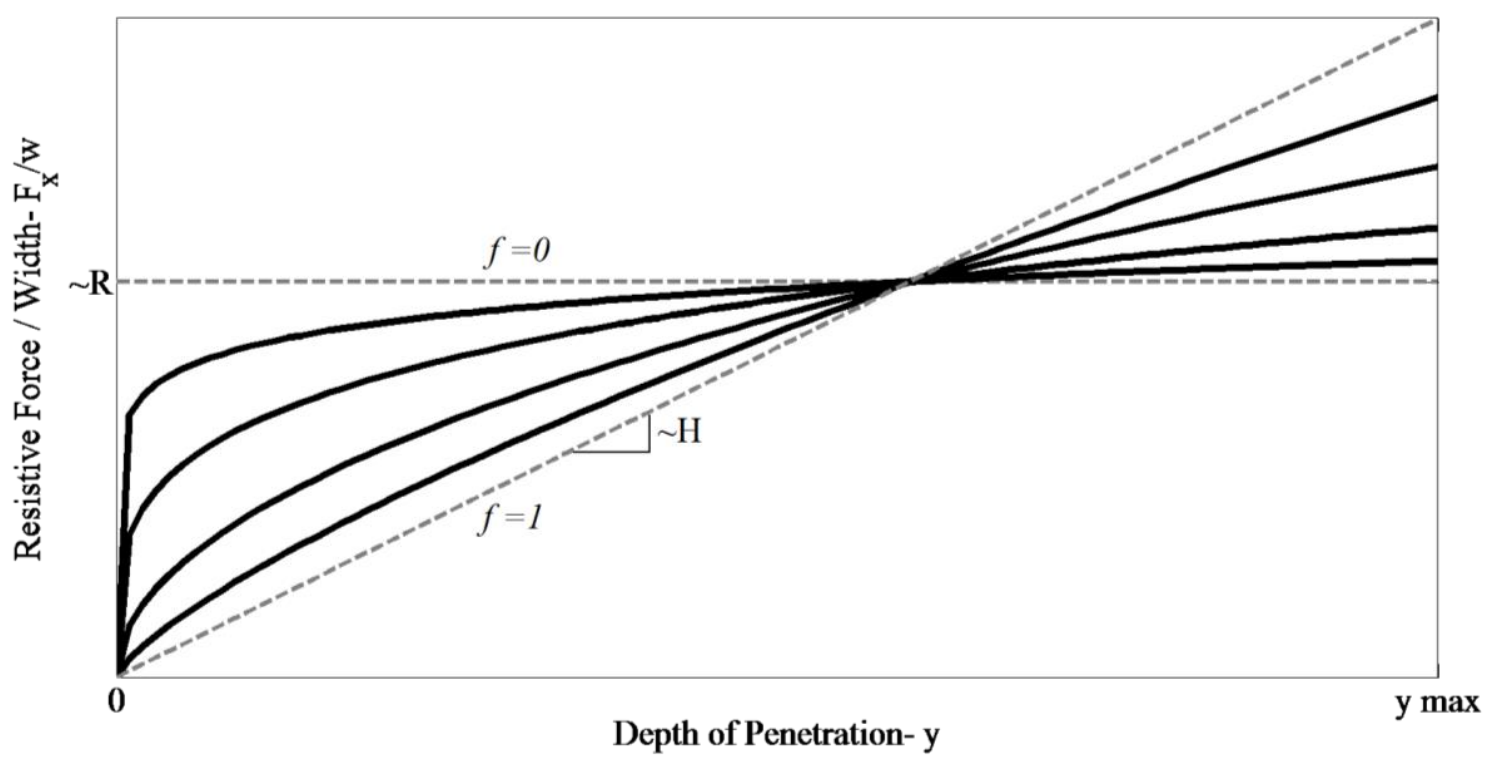

Fig. 5. Horizontal force simplified model for various f exponents. In the following example, $R=6 \mathrm{~kJ} / \mathrm{m}^{2}$, $H=1 \mathrm{GPa}$.

As can be seen in Fig. 5, the extreme values of $f$ gives the two extreme modes: $f=1$ presents ideal ductile mode- Eq. (14) while $f=0$ presents ideal brittle mode, Eq. (17). The shape of graphs protruded in Fig. 5 is very similar to plots of $F_{x} / w$ versus depth of cut received for orthogonal cutting [21].

Assuming linear relation between depth of penetration, $y$ and length of the scratch, $x$, the work done by the particle horizontal movement $F_{x} d x$ is proportional to $F_{x} d y$ - the area beneath the graphs in Fig. 5. The value of the exponent $f$ should be governed by minimum work criterion, thus $f$ is a function of the ratio of the material fracture toughness $(R)$ to the hardness of the target material $(H)$ and the maximum penetration depth $\left(y_{\max }\right)$. The depth of penetration is a derived value, controlled by the dynamic properties of the impact. Hence, we define the effective particle diameter as the typical length scale of the indentation, for a given impingement angle and velocity.

$$
f=f\left(R / H, d_{\text {eff }}\right)
$$

The dimensions of $R / H$ are [m] and the dimensions of $d_{\text {eff }}$ are [m], hence division of these two parameters gives a non-dimensional number: $R / H \cdot d_{\text {eff. }}$ This number is much similar to the parameter found by Hutchings [13]: $K_{c}^{2} / r \cdot H^{2}$, where $r$ is the particle radius and $K_{c}\left[\mathrm{MPa} \cdot \mathrm{m}^{1 / 2}\right.$ ] is the critical stress intensity factor around the crack tip that can be related to the fracture toughness by $R=K_{c}^{2} / E$, where $E$ is the young modulus. Hutchings [13] remarked that when this number is small the material response is dominated by fracture mechanism, which is the same trend as our model predicts. Taking into account the relatively shallow penetration of particles in erosion process, the use of effective diameter (which is a function of the actual diameter and the particle shape) is more physical and takes into account the effect of particle sharpness. The sharper particles are (smaller $d_{e f f}$ ) and more ductile the target material is (larger $R / H$ ), the larger value of $f$ received- 
the erosion mechanism trends into ploughing. One can notice in Fig. 5 that indeed if $y_{\max }$ (proportional to $d_{e f f}$ ) is small, $f=1$ will yield minimum energy, while for large $y_{\max }$, minimum energy obtained for lower $f$.

Substituting (19) in (12) and integrating over $x$ (assuming $y \in\left[0, y_{\max }\right]$ is linear in $x \in[0, L / 2]$ ) yields:

$$
\int_{0}^{L} F_{x} d x=w \cdot L\left(a_{1} \cdot H \cdot y_{\max }\right)^{f}\left(a_{2} \cdot R\right)^{1-f} /(1+f)
$$

Than substituting (21) back to (12), the scratch length, $L$, is obtained:

$$
L=c_{2}(1+f) \frac{m_{p} U_{x 0}^{2}}{w\left(H \cdot y_{\max }\right)^{f} R^{1-f}}
$$

where, $c_{2}=\left(1-e_{x}^{2}\right) / 2 a_{1}^{f} a_{2}^{1-f}$. For further simplicity from now forward it is assumed that $c_{2}$ is not material dependent. In low impingement angles (under $10^{\circ}$ ), effects of particles detachment due to rotation are more likely to occur, especially with particles with irregular shapes. Hence, the horizontal restitution term $1-e_{x}^{2}$, should be lower. While this effect is very hard to model, it can be accounted for in a numerical way by adding a damping function which will lower the erosion in this range. The function $f_{\text {damp }}(\alpha)=1-\exp \left(-200 \alpha^{2}\right)$ was found to be suitable for all materials examined.

Assigning $y_{\max }(11)$ and $L(21)$ into Eq. (2):

$$
\Delta Q_{C}=c_{3}(1+f)\left(1-\exp \left(-200 \alpha^{2}\right)\right) \frac{m_{p}^{1+(1-f) / 2}}{\left(\pi d_{e f f}\right)^{(1-f) / 2} H^{(1+f) / 2} R^{1-f}} \cdot U_{x 0}^{2} U_{y 0}^{1-f}
$$

where, $c_{3}=c_{2} \cdot c_{1}$.

Writing the components of the particle velocity with regard to the impingement angle $\alpha$ yields:

$$
\Delta Q_{C}=c_{3}(1+f)\left(1-\exp \left(-200 \alpha^{2}\right)\right) \frac{m_{p}^{1+(1-f) / 2}}{\left(\pi d_{e f f}\right)^{(1-f) / 2} H^{(1+f) / 2} R^{1-f}} \cdot U_{0}^{3-f} \cos ^{2}(\alpha) \sin ^{1-f}(\alpha)
$$

The effective particle diameter may be related via particle shape factor, $d_{\text {eff }}=\eta \cdot d_{p}$, where the value of $\eta$ lays between 0 to 1 and is larger the more spherical particle the particle is. The particle mass can be approximated by $m_{p}=\pi \rho_{p} \cdot d_{p}^{3} / 6$. Thus:

$$
\left(\frac{m_{p}}{\pi d_{e f f}}\right)^{(1-f) / 2} \propto\left(\frac{\rho_{p} d_{p}^{2}}{\eta}\right)^{(1-f) / 2}
$$


Finally, the volume loss per particle mass in 'cutting' mechanism can be calculate by:

$$
\frac{\Delta Q_{C}}{m_{p}}=C_{C}(1+f)\left(1-\exp \left(-200 \alpha^{2}\right)\right) \frac{\left(\rho_{p} d_{p}^{2} / \eta\right)^{(1-f) / 2}}{H^{(1+f) / 2} R^{1-f}} \cdot U_{0}^{3-f} \cos ^{2}(\alpha) \sin ^{1-f}(\alpha)
$$

Eq. (26) introduces a newly developed 'cutting' mechanism model for erosion rate. Examination of the developed model can lead to further insight on the 'cutting' mechanism in erosion:

- The velocity exponent in the model lies between 3 for fracture governed erosion and 2 for ploughing erosion. This result fits well with dimensional analysis:

For both mechanisms the energy available for erosion is the particle kinetic energy which is proportional to $U_{0}^{2}$.

For fracture governed erosion, the energy required for material removal is proportional to the area of the scratch: $E \propto l^{2}$, where $l$ is a characteristic length of the scratch. Hence,

$l^{2} \propto U_{0}^{2}$. Consequently, the volume of material removal has a cubic relation with the velocity: $l^{3} \propto U_{0}^{3}$.

For ploughing erosion, the energy required for material removal is proportional to the removed volume: $E \propto l^{3}$. Hence, the removed volume is proportional to the kinetic energy:

$l^{3} \propto U_{0}^{2}$.

It was observed by Sheldon \& Kanhere [24] that annealed material have lower velocity exponent than work hardened material. This supports the claim that ductility of the target material (given by $R / H$ ratio), which changes the material removal mechanism is the dominant factor governing the velocity exponent.

- The power $f$ presents the effectiveness of the ploughing erosion process. The larger $f$ is, the process trends into ideal ductile model. Assigning $f$ its maximum value, $f=1$, the volume loss due to 'cutting' mechanism results in:

$$
\Delta Q_{C}=C_{C} \cdot \frac{m_{p}}{H} \cdot U_{0}^{2} \cos ^{2}(\alpha)
$$

which is essentially the Finnie's [3] model for ploughing particle.

- For the effect of target material hardness, the relation for erosion in 'cutting' mechanism is:

$$
\Delta Q_{C} \propto \frac{1}{H^{0.5 \div 1}}
$$

Finnie's [11] experimental data for the relation between target material hardness and erosion resistance to 'cutting' mechanism (represented by $1 /$ [erosion rate] in impingement angle of $20^{\circ}$ ) is presented in Fig. 6. As can be seen, according to Finnie's [11] experiments, the relation of the volume of material removal to target material hardness is:

$$
\Delta Q_{C} \propto \frac{1}{H^{0.6 \div 1}}
$$

which agrees with the range in our model. Interesting feature, one can see in Fig. 6 is that the exponent for harder material is lower than for softer ones. This feature is predicted in the model; 
harder material have lower $f$ exponent, due to ascent in their brittleness, hence the exponent of $H$ is lower. From mechanistic point of view this trend is very reasonable due to the growing dominance of fracture mechanism at the expanse of ploughing mechanism with the growth in the hardness.

Further experiments conducted by Arabnejad et al. [10] yielded:

$$
\Delta Q_{C} \propto \frac{1}{H^{0.5}}
$$

Hence the prediction of the developed model is supported by the experimental findings of both Finnie [11] and Arabnejad et al. [10].

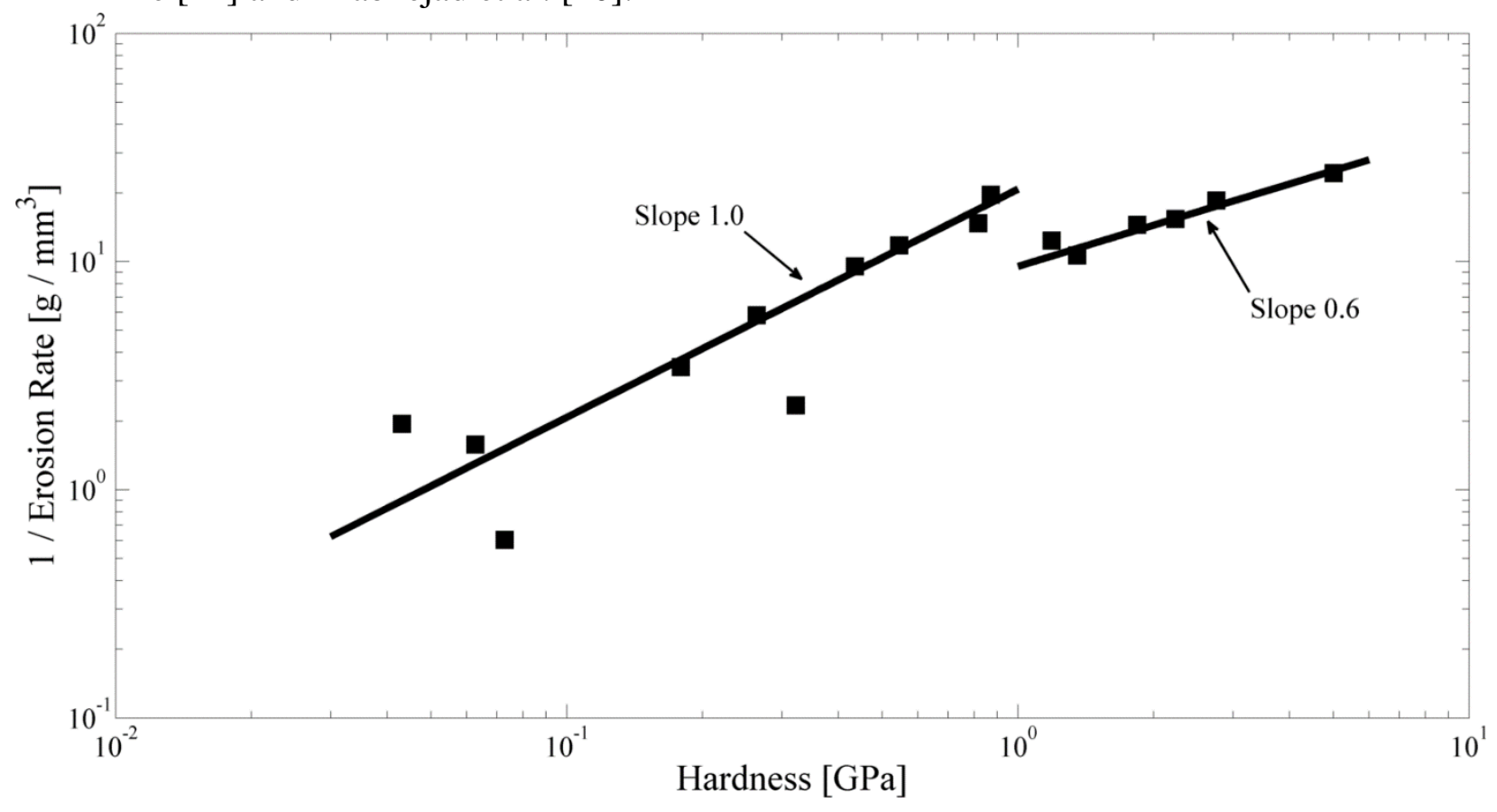

Fig. 6. Inverse erosion rate as a function of target material Vickers hardness. Impingement velocity is $76.2 \mathrm{~m} / \mathrm{s}$, impingement angle is $20^{\circ}$. Abrasive particles are $250 \mu \mathrm{m}$ SiC. From Finnie's paper [11].

\section{2. 'Deformation' mechanism erosion}

For erosion by 'deformation' mechanism, Hutchings [6] model, which accounts for normal impingement angle erosion, is adopted with a few modifications. For the sake of completeness this model is presented here.

'Deformation' erosion is caused by crack propagation due to repeated plastic deformation, hence the model should incorporate fatigue fracture model, such as, Coffin-Manson criterion for low cycle fatigue. The Coffin-Manson criterion relates the number of loading cycles for fracture $N_{f}$ to the amplitude of plastic strain at each cycle $\Delta \varepsilon$ :

$$
\varepsilon_{f}=2 \Delta \varepsilon \cdot N_{f}^{b}
$$


where $\varepsilon_{f}$ is the fatigue ductility coefficient and $b$ is an empirical exponent lays in the range of $0.5 \div 0.7$ for metals and steels [25].

The strain amplitude is given by the square root of the relative change in the surface area:

$$
\Delta \varepsilon=\sqrt{\left(A_{y f}-A_{y 0}\right) / A_{y 0}} \propto y_{\max } / D
$$

where $y$ and $D$ are the depth and diameter of indentation, respectively. Using the relation in Eq.(4)

$$
\Delta \varepsilon \propto \sqrt{y_{\max } / d_{e f f}}
$$

According to Hutchings [6] methodology, a number of particles impacts $N_{f}$ is required to remove an 'elementary volume' of material- $\Delta Q_{E}$, which is the volume of indentation produced by a particle impact. Therefore, the amount of material removal at a single impact is the product of division of the elementary volume in $N_{f}$ :

$$
\Delta Q_{D} \propto \frac{\Delta Q_{E}}{N_{f}}
$$

By assuming all of the normal velocity kinetic energy of the particle transforms into plastic deformation at a constant flow stress, the elementary volume of the indentation is expressed:

$$
\Delta Q_{E}=\frac{m_{p} U_{0 y}^{2}}{2 H}
$$

Combining Eq. (11), (31), (33), (34) and (35), yields:

$$
\Delta Q_{D}=C_{D} \cdot \frac{m_{p}{ }^{1+1 / 4 b}}{\varepsilon_{f}{ }^{1 / b} H^{1+1 / 4 b} d_{e f f}{ }^{3 / 4 b}}\left(U_{0} \sin \alpha\right)^{2+1 / 2 b}
$$

Making the same substitution as in Eq. (26), yields:

$$
\frac{\Delta Q_{D}}{m_{p}}=C_{D} \cdot \frac{\rho_{p}{ }^{1 / 4 b}}{\eta^{3 / 4 b} \varepsilon_{f}{ }^{1 / b} H^{1+1 / 4 b}}\left(U_{0} \sin \alpha\right)^{2+1 / 2 b}
$$

where $b \sim 0.5 \div 0.7$, the velocity exponent lays in the range $2.7 \div 3$, which fits reasonably well with experimental data [6].

By neglecting mutual effect of the two mechanisms of erosion, the total erosion is the sum of the two: $\Delta Q=\Delta Q_{D}+\Delta Q_{C}$

$$
\begin{aligned}
\frac{\Delta Q}{m_{p}}= & C_{D} \cdot \frac{\rho_{p}{ }^{1 / 4 b}}{\eta^{3 / 4 b} \varepsilon_{f}{ }^{1 / b} H^{1+1 / 4 b}}\left(U_{0} \sin \alpha\right)^{2+1 / 2 b}+ \\
& C_{C}(1+f)\left(1-\exp \left(-200 \alpha^{2}\right)\right) \frac{\rho_{p}{ }^{(1-f) / 2} d_{p}{ }^{1-f}}{\eta^{(1-f) / 2} H^{(1+f) / 2} R^{1-f}} \cdot U_{0}^{3-f} \cos ^{2}(\alpha) \sin ^{1-f}(\alpha)
\end{aligned}
$$




\section{Effect of $f$ exponent on prediction of maximum erosion angle}

As mentioned earlier, the main problem with Huang et al. [9] model is its incapability of predicting the right maximum erosion angle. In this section, the advantage of the newly developed model is presented by introducing the $f$ exponent which governs the maximum erosion angle. Predictions from the developed model are validated with experimental data in respect to the effect of impingement angle. Our model prediction (Eq.(38)) for the experimental results of Finnie (from Huang et al. [9] paper) is presented in Fig. 7 along with the original Huang et al. [9] model prediction. The experiments were conducted using $250 \mu \mathrm{m}$ diameter $\mathrm{SiC}$ particles $\left(\rho_{p}=3200 \mathrm{~kg} / \mathrm{m}^{3}\right.$ , $\eta=0.3$ ) at impact velocity of $107 \mathrm{~m} / \mathrm{s}$ for three target materials: aluminum, copper and SAE-1055 steel. The experimental data was correlated to obtain the model's constants, where all the unknown target materials properties were incorporated in the coefficients $\tilde{C}_{D}=C_{D} / \varepsilon_{f}{ }^{1 / b} H^{1+1 / 4 b}$, $\tilde{C}_{C}=C_{C} / H^{(1+f) / 2} R^{1-f}$ (listed in table 1) and $b$ was set to 0.6. This yields the following simplified equation for erosion rate:

$$
\frac{\Delta Q}{m_{p}}=\tilde{C}_{D} \frac{\rho_{p}^{0.42}}{\eta^{1.25}} \cdot\left(U_{0} \sin \alpha\right)^{2.83}+\tilde{C}_{C}(1+f)\left(1-\exp \left(-200 \alpha^{2}\right)\right) \frac{\rho_{p}^{(1-f) / 2} d_{p}^{1-f}}{\eta^{(1-f) / 2}} \cdot U_{0}^{3-f} \cos ^{2}(\alpha) \sin ^{1-f}(\alpha)
$$

The validation with Finnie's data was made in order to prove that the model can predict the effect of impingement angle on the erosion rate for various target materials and to exhibit the prominent advantage of the newly derived model, compared to the Huang et al. [9] model. The better agreement of the newly developed model with the experimental data can be seen in in Fig. $7 \mathrm{~b}$ and $7 \mathrm{c}$, where the target materials were copper and aluminum respectively. The Huang et al. [9] model predicts almost identical maximum erosion angle for all three target materials (which fits well only to the results for 1055 steel, Fig. 7a), while our model predicts an accurate angle for all three materials. This is due to the $f$ exponent that was introduced which governs the maximum erosion angle. The maximum erosion angle is a function of the erosion mechanism; for ductile materials such as aluminum, the ratio of the fracture energy to plastic deformation energy is large, hence the ploughing process is preferred and the erosion rate is higher in shallower impingement angles. The $f$ exponent depends upon this ratio and therefore governs the maximum erosion angle. Values of $f$ received are $0.55,0.80$ and 1.00 for SAE-1055 steel, copper and aluminum respectively. 

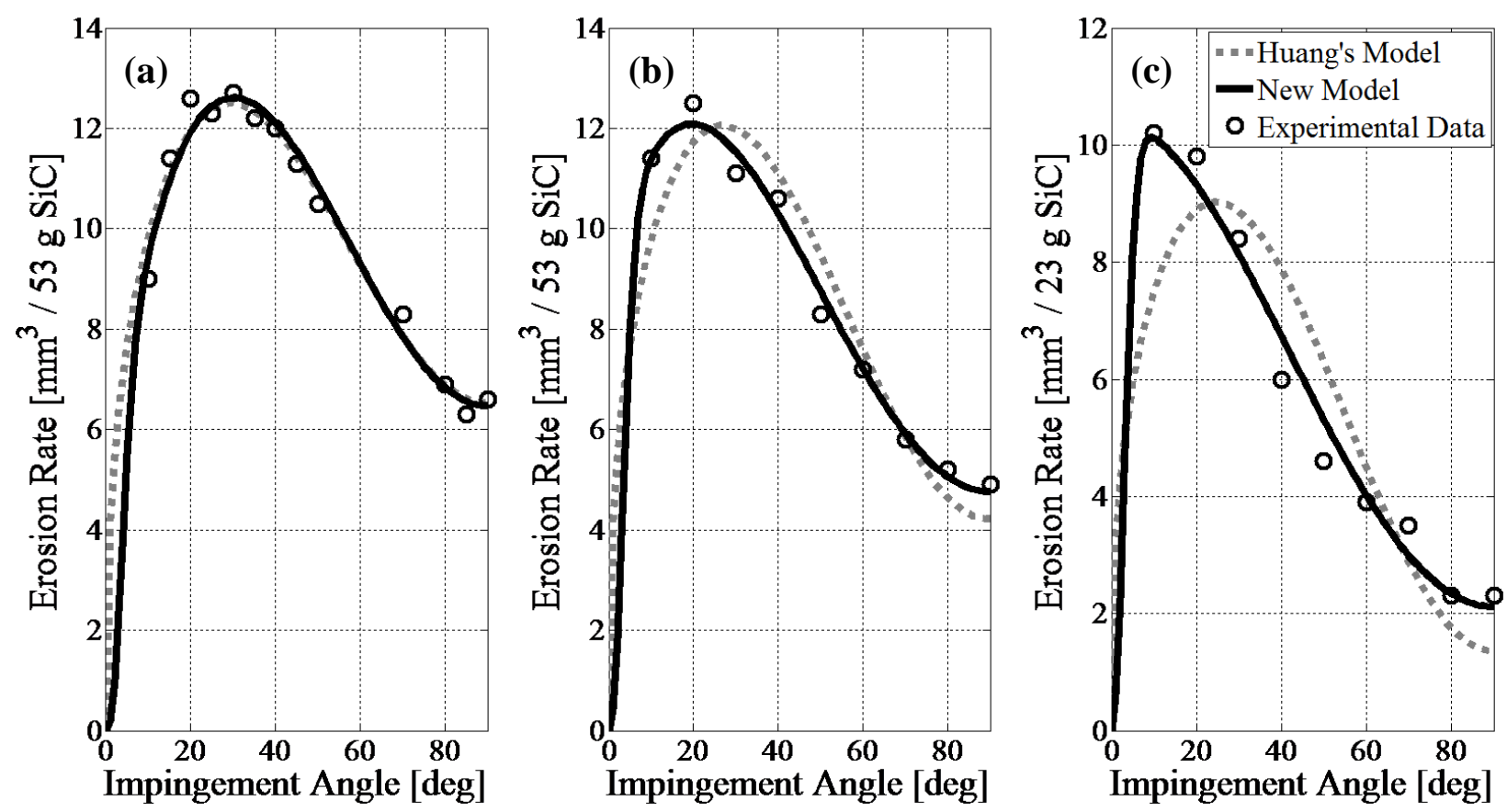

Fig. 7. Erosion rate as a function of impingement angle- Huang et al. [9] and new developed models vs. experimental results of Finnie (from Huang et al. [9] paper). Target materials are (a)SAE-1055, (b)copper and (c)aluminum. Impingement velocity is $107 \mathrm{~m} / \mathrm{s}$. Abrasive particles are $250 \mu \mathrm{m}$ Sic.

Table 1- Model coefficients for Finnie's Experiments

\begin{tabular}{lccc}
\hline Target Material & $f$ & $\tilde{C}_{D} \times 10^{6}$ & $\tilde{C}_{C}$ \\
\hline Aluminum & 1 & 1.245 & 0.02034 \\
Copper & 0.80 & 1.640 & 0.01242 \\
SAE-1055 & 0.55 & 1.941 & 0.01434
\end{tabular}

\section{The effect of target material properties}

Examination of the model prediction for the effect of the target material properties was made using experimental data obtained by Oka et al. [15],[16]. The erosion experiments were conducted using $326 \mu \mathrm{m}$ average diameter $\mathrm{SiO}_{2}$ abrasive particles $\left(\rho_{p}=2600 \mathrm{~kg} / \mathrm{m}^{3}, \eta=0.3\right)$. The impact velocities used in the experiments were of 100-104 m/s. The experimental data was correlated by iterative least square method to obtain the model's constants: $C_{D}, C_{C}, f$, where the unknown parameters of the material fatigue ductility coefficient, $\varepsilon_{f}$, were incorporated in the coefficient $C_{D}$. The materials Vickers hardness was given by Oka et al. [15],[16] and their fracture toughness was evaluated from literature [26]-[30] of similar materials. Table 2 lists the target material properties used in the model. The fatigue ductility exponent- $b$, was set as 0.6 for ductile materials $\left(H / R<20 \mathrm{~mm}^{-1}\right)$ and 0.5 for more brittle materials [25]. 
Table 2- Target material properties for Oka's experiments

\begin{tabular}{lcccc}
\hline Target Material & $\mathrm{Hv}[\mathrm{GPa}]$ & $\mathrm{K}_{\mathrm{C}}\left[\mathrm{MPa} \cdot \mathrm{m}^{1 / 2}\right]$ & $\mathrm{R}\left[\mathrm{kJ} / \mathrm{m}^{2}\right]$ & $\mathrm{E}[\mathrm{GPa}]$ \\
\hline Lead & 0.20 & - & $75^{(\mathrm{a})}$ & 20 \\
Aluminum & 0.40 & - & $100^{(\mathrm{b})}$ & 70 \\
Iron & 1.50 & - & $80^{(\mathrm{c})}$ & 160 \\
Grey cast iron & 3.00 & $35^{(\mathrm{d})}$ & $10^{(\mathrm{f})}$ & 130 \\
Austenitic steel S2 & 4.41 & $65^{(\mathrm{e})}$ & $20^{(\mathrm{f})}$ & 205 \\
Martensitic steel S3 & 4.46 & $65^{(\mathrm{e})}$ & $20^{(\mathrm{f})}$ & 205 \\
Martensitic steel S4 & 4.87 & $65^{(\mathrm{e})}$ & $20^{(\mathrm{f})}$ & 205 \\
Martensitic steel S5 & 6.50 & $65^{(\mathrm{e})}$ & $20^{(\mathrm{f})}$ & 205
\end{tabular}

(a) Atkins [23] after Kobayshi \& Thomsen [26]

(b) Atkins [27]

(c) Salzbrenner [28]; value for ductile iron having tensile strength of $480 \mathrm{MPa}(\mathrm{Hv} \approx 1.5 \mathrm{GPa})$

(d) Hornbogen [29]; value for GGG80 grey cast iron, $\mathrm{HB}=260(\mathrm{Hv} \approx 2.7 \mathrm{GPa})$

(e) Ledbetter [30]; value for stainless steel costum 455 , tensile strength $=1600 \mathrm{MPa}(\mathrm{Hv} \approx 4.8 \mathrm{GPa})$

(f) Values derived using the relation: $\mathrm{R}=\mathrm{K}_{\mathrm{C}}{ }^{2} / \mathrm{E}$

\subsection{Impingement angle dependence}

The newly developed model prediction in comparison to the experimental data for the erosion of various metals and cast iron and for the erosion of various stainless steels is presented in Figs. 8 and 9, respectively. As can be seen, fairly good agreement between model prediction and experimental data for wide range of target materials is obtained. Note that the hardness of the target material is not the only factor affecting the extent of erosion, such in the case of iron versus cast iron and in the case of the various stainless steels. The transition in the maximum erosion angle as a function of the material ductility is noticeable in Fig. 8: $10^{\circ}$ for lead to $40^{\circ}$ for grey cast iron. This transition is due to erosion mechanism change from ductile to brittle mode governed by the ratio of fracture toughness to hardness. The more brittle the material is (higher $H / R$ ), it has higher maximum erosion angle and the ratio of their peak erosion to erosion at normal impact is lower. Both of these features show lesser ploughing efficiency and a stronger trend to fracture mechanism. The larger the impingement angle is, the larger the extent of deformation required for the particle to plough through the material. The increase in the required plastic deformation energy with the increase in the impingement angle is larger for harder materials. Thus, a transition to fracture mechanism is more likely to occur. The energy required to extend a fracture is less dependent on the depth of penetration than the ploughing energy. Hence, growing dominance of fracture mechanism is shown by larger maximum erosion angle. 


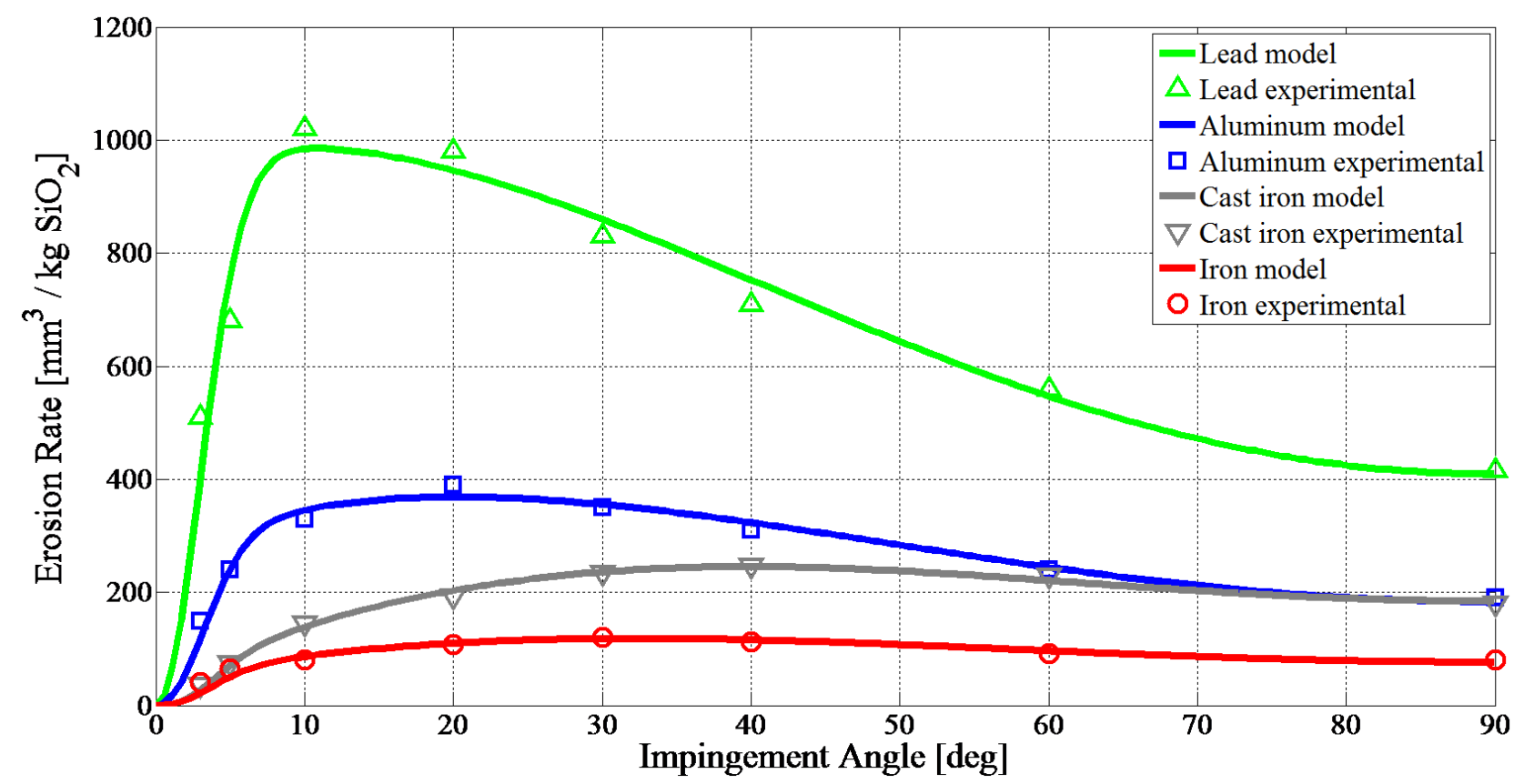

Fig. 8. Erosion rate as a function of the impingement angle. Model prediction vs. Oka et al. [15] experimental results.

The goodness of the fit between the new model and the experiments is about the same as Oka et al. [16] model. Nevertheless, the developed model is only semi-empirical, while Oka et al. [16] model is fully-empirical. Hence, the empirical coefficients and exponents we received through the experiments should have a physical interpretation and their values for other target materials can be deduced by using correlations to known target material properties. This feature will be discussed in the next section.

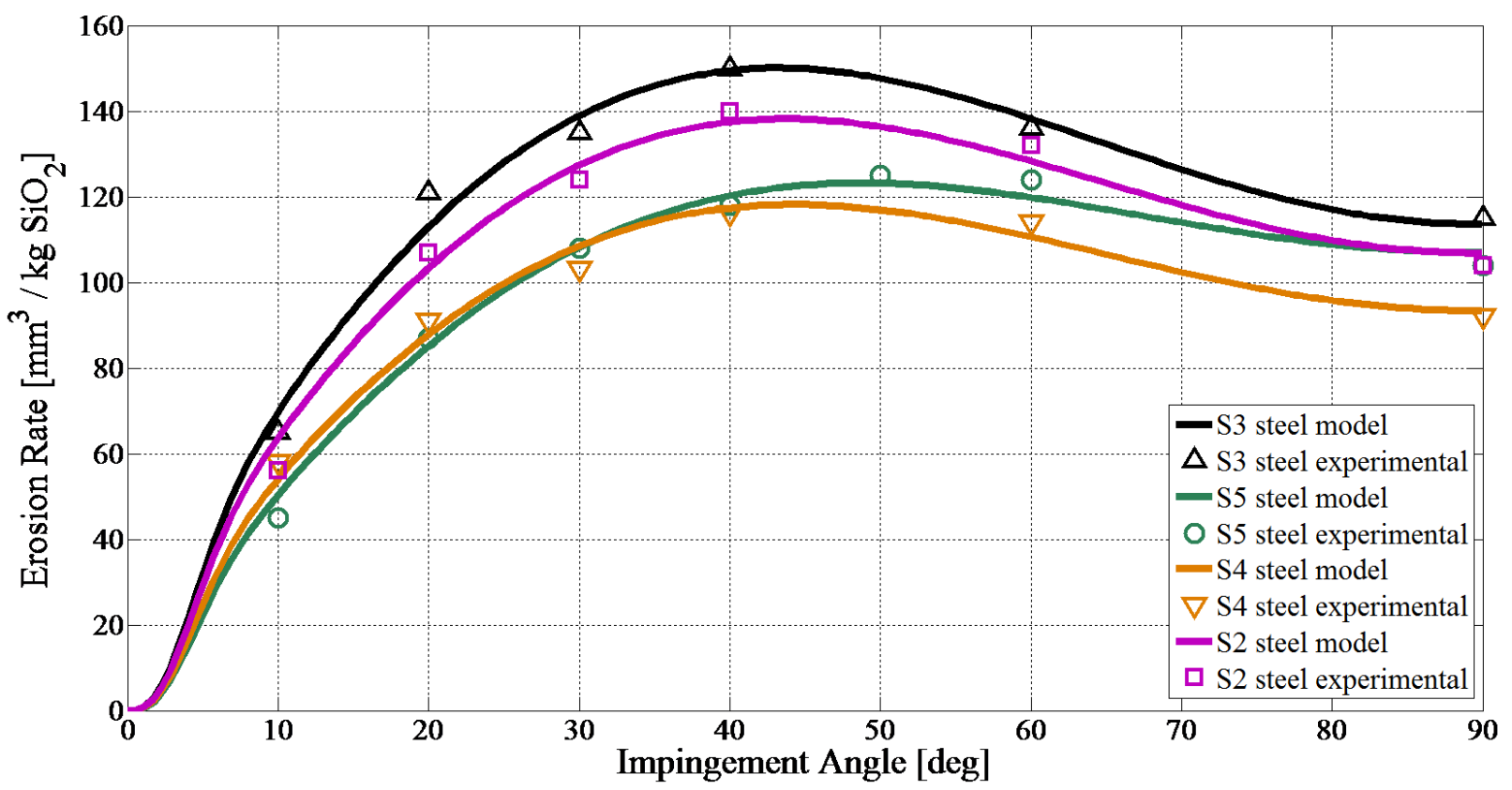

Fig. 9. Erosion rate as a function of the impingement angle. Model prediction vs. Oka et al. [16] experimental results. 


\subsection{Model empirical coefficients}

Table 3- Model coefficients for Oka's experiments

\begin{tabular}{lccc}
\hline Target Material & $f$ & $C_{D}$ & $C_{C}$ \\
\hline Lead & 0.95 & 0.00448 & 0.01168 \\
Aluminum & 0.80 & 0.00537 & 0.01240 \\
Iron & 0.55 & 0.01459 & 0.01588 \\
Grey cast iron & 0.25 & 0.09473 & 0.01420 \\
Austenitic steel S2 & 0.20 & 0.08958 & 0.01373 \\
Martensitic steel S3 & 0.20 & 0.09686 & 0.01512 \\
Martensitic steel S4 & 0.25 & 0.09083 & 0.01237 \\
Martensitic steel S5 & 0.15 & 0.16050 & 0.01371
\end{tabular}

The newly developed model coefficients that were found are listed in Table 3. In order to have a more universal model we wish to relate the coefficients $C_{D}, C_{C}$ and $f$ to measurable target material properties. Oka et al. [15],[16] suggested an empirical model and correlated his model coefficients to the material property of hardness alone. By dimensional analysis it can be shown that if hardness is the sole target material property that governs the erosion process, then the volume of eroded material should be: $\Delta Q \sim m U_{0}^{2} / H$. Hence, no reason to receive velocity exponent larger than 2 , which is contradictory to experimental findings $\left(\Delta Q \sim U^{2 \div 3}\right)$. Moreover, simulations of angular and spherical abrasive particles impact against ductile target material conducted by some researchers [20],[31],[32], which took into account only the effect of plastic deformation and friction, yielded results that the maximum indentation volume is at normal impact, contradictory to experimental findings of erosion. This led us to the conclusion that fracture mechanism have to be accounted for and therefore fracture toughness must be included in the form of the ratio of $H / R$. Hence, the developed model empirical coefficients was correlated to this ratio.

The $f$ exponent which governs the transition between ductile to brittle modes is not a real material property, but present a model for the transition as depicted in Eq. (19), from which the work of the particle can be derived (Eq. (21)). For given target materials, the mode of material removal should be governed by minimum energy criterion. Thus, differentiating Eq. (21) in $f$ and equating to zero gives the $f$ exponent that will yield the minimum work of the particle:

$$
f=\frac{1}{\ln \left(a H \cdot y_{\max } / b R\right)}-1
$$

The minimum work criterion suggests that $f$ have inverse logarithmic relation with $H / R$. Hence such a function was fitted to the $f$ exponents received from the experiments. Fig. 10 presents the results of this fitting. The equation for $f$ which was received is:

$$
f=\frac{14}{\ln (537 H / R)}-1
$$


where the units of $H[\mathrm{MPa}] / R\left[\mathrm{~kJ} / \mathrm{m}^{2}\right]$ are $\mathrm{mm}^{-1}$.

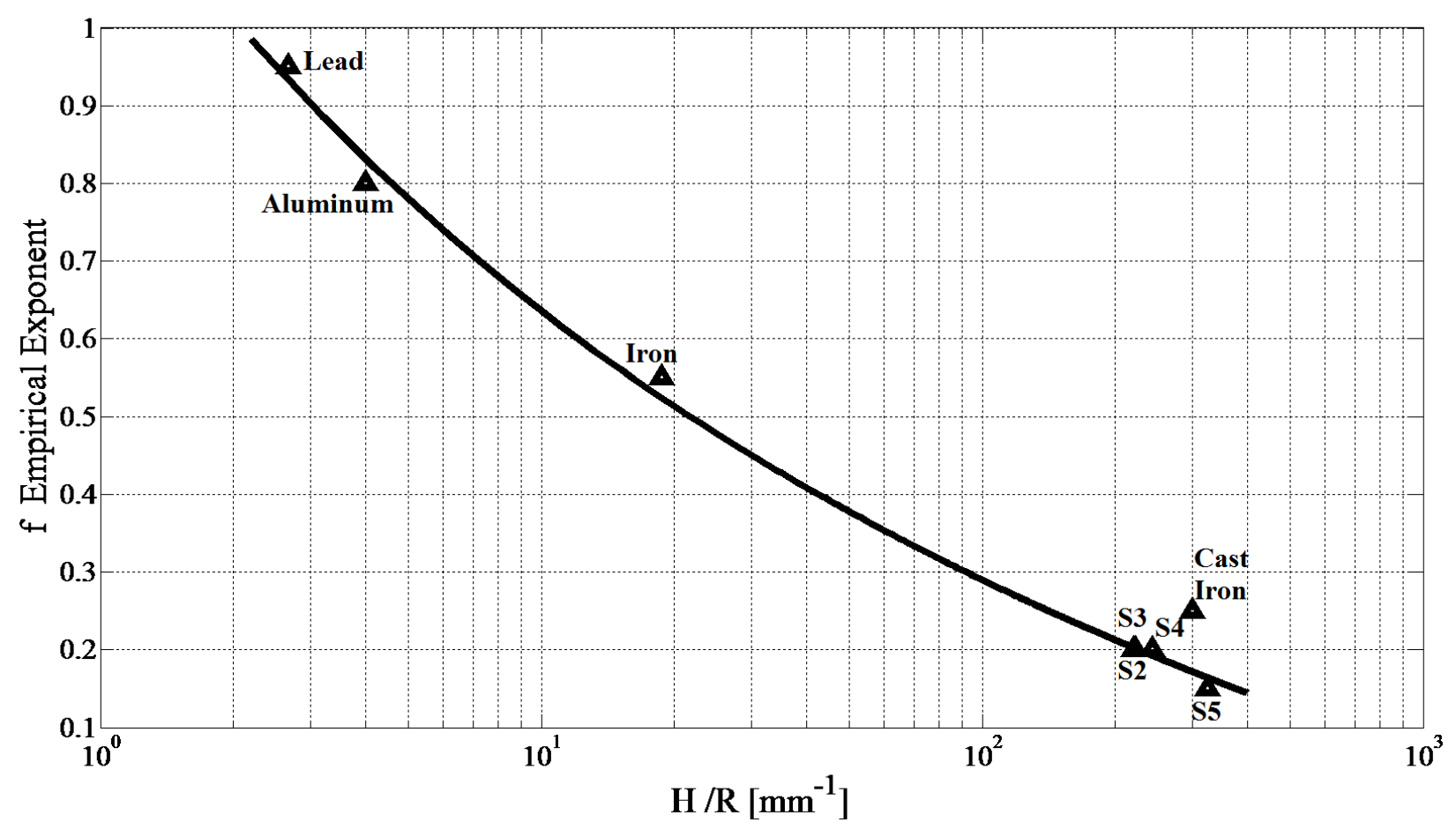

Fig. 10. Empirical exponent, $f$, as a function of the ratio of target material hardness to fracture toughness. Values calibrated from Oka et al. [15],[16] experiments and fitted inverse logarithmic function.

The inverse logarithmic trend is noticeable in Fig. 10, where $f$ descends sharply at low $H / R$ and trends into relatively constant value of about 0.2 at $H / R \sim 200 \div 350 \mathrm{~mm}^{-1}$. This model has a minimum $H / R$ limitation of $2 \mathrm{~mm}^{-1}$. More ductile materials will receive values of $f$ greater than 1 , which are not physical values. However, materials of such ductility (very soft metals or rubber) can be deduced to have $f$ value of 1 , meaning fracture erosion mechanism does not exist for these kind of materials.

Fig. 11 presents the $C_{D}$ empirical coefficient, which is the factor of the 'deformation' erosion mechanism. The coefficient $C_{D}$ is inversely proportional to $\varepsilon_{f}$, the fatigue ductility coefficient from Coffin-Manson criterion. Due to its dependence on the ductility, some researchers [25] tried to relate $\varepsilon_{f}$ to the ratio of hardness to Young modulus. While, this relation can be valid for small plastic deformation, in erosion process, where significant plastic deformations take place the use of $R / H$ as a measure for the material ductility is better. The length scale given by $R / H$ should give the critical crack length for fracture. The values of $C_{D}$ versus $H / R$ are presented in Fig. 11 and the relation that was found is:

$$
C_{D}=0.002(H / R)^{0.7}
$$


$C_{D}$ rises with $H / R$ because it is proportional to the material brittleness, i.e. inversely proportional to $\varepsilon$, which decrease with $H / R$. The harder and the less fracture tough the material is, it is more vulnerable to cracks propagation, thus the deformation mechanism is more significant. The very clear trend of $C_{D}$ is very important because it is suggests that $H / R$ is in fact its governing parameter. The largest error received is about $30 \%$ for the most brittle material- martensitic steel S5.

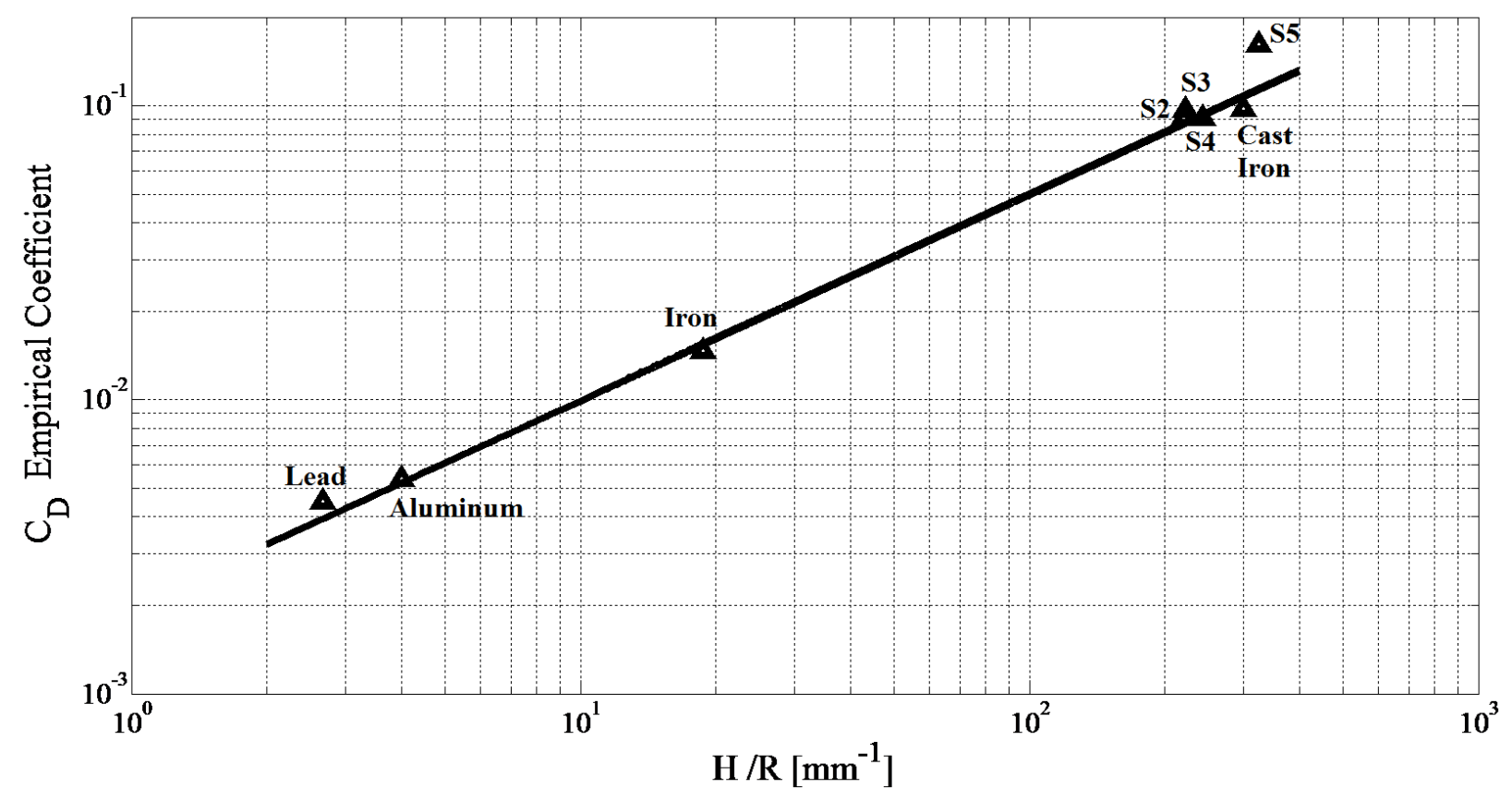

Fig. 11. Empirical coefficient, $C_{D}$ as a function of the ratio of target material hardness to fracture toughness. Values calibrated from Oka et al. [15],[16] experiments and fitted function.

The significant erosion of S5 in the 'deformation' mechanism is noticeable in Fig. 9, where its ratio of erosion in normal impingement angle to maximum erosion is almost equals to unity, which is much larger than other stainless steels that were tested. Another point which diverged slightly from the trend is the second most brittle material- grey cast iron (20\% error). The divergence of these two materials suggests that the model have an error in the range of very brittle materials $(H / R>300$ $\mathrm{mm}^{-1}$ ). However, the error is not large, taking into account that this model is valid for very large range of materials and probably will be reduced if a more precise measure of the fracture toughness will be taken.

The 'cutting' mechanism factor, $C_{C}$, is presented in Fig. 12 as a function of $H / R$. It can be noticed that $C_{C}$ presents no ascending or descending trend. An average value of 0.0136 was received within an error range of $15 \%$. Thus we can assume $C_{C}$ as a model constant and the assumption we made in Eq. (22) is justified. Analysis of the factors incorporated in $C_{C}$ leads to ambivalent conclusion regarding the trend that should be received. On the one hand, the restitution ratio $\left(1-e_{x}^{2}\right)$ should rise with the ductility (as was shown in Sundararajan [33] work), making more energy available for material removal; On the other hand $c_{1}$, the ratio of deformed volume to volume of material removal is inversely related to the ductility and also depends on the mechanism of material removal- ploughing or fracture. For this reason, the constant value received for $C_{C}$ is quite reasonable. The fact that $C_{C}$ shows no dependent of $H / R$ (and consequently is independent of $f$ 
exponent) suggests that the effect of target material properties on the 'cutting' erosion was modelled accurately and hence supports the validity of the developed model.

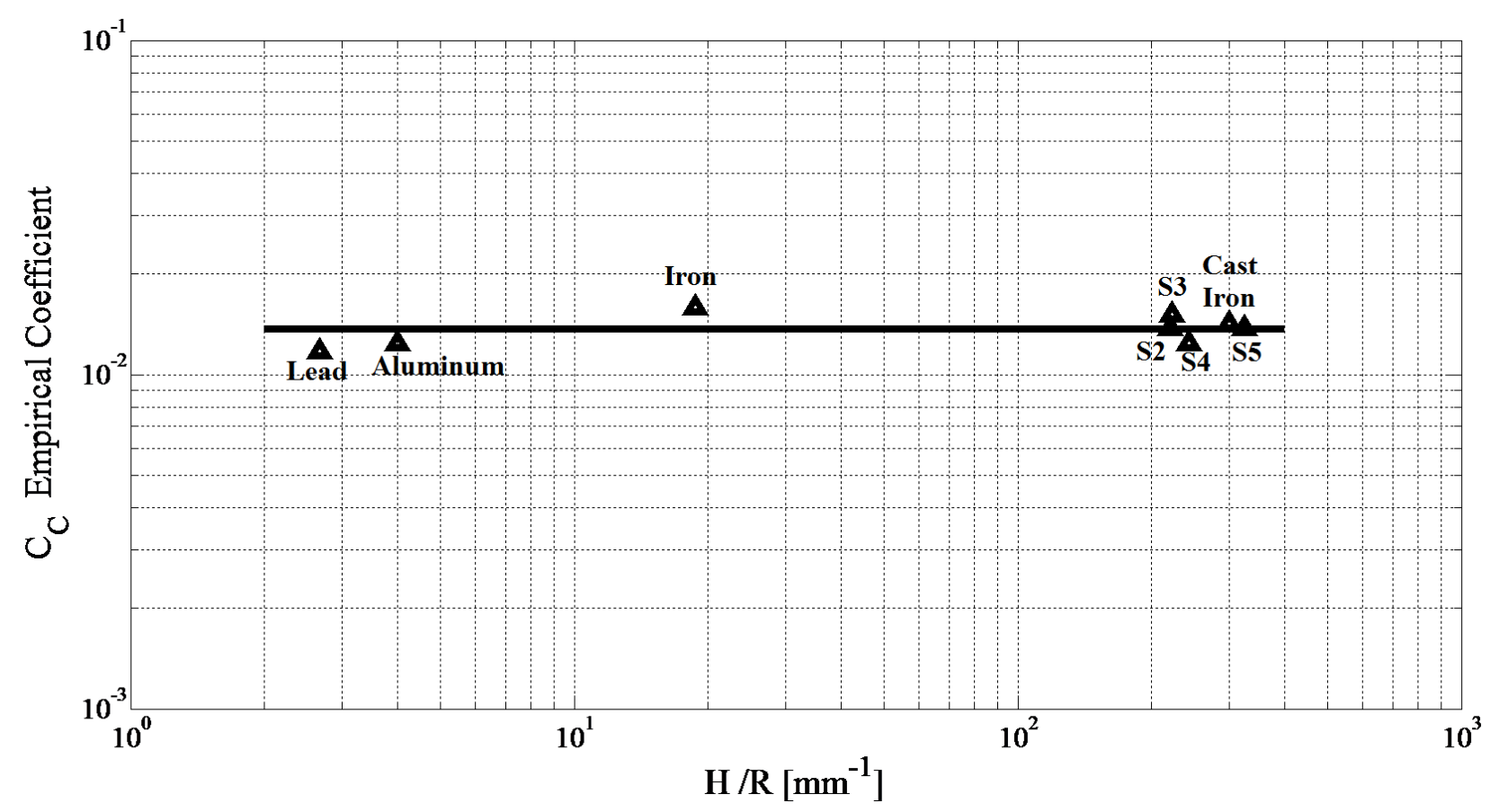

Fig. 12. Empirical coefficient, $C_{C}$ as a function of the ratio of target material hardness to fracture toughness. Values calibrated from Oka et al. [15],[16] experiments and average value line.

\section{Effect of Impact Velocity}

The developed model was validated using experimental results of erosion by particle impacts at very high speed- $100 \mathrm{~m} / \mathrm{s}$. This velocity magnitude is standard for experimental purposes due to the high erosion rate which makes the measurement easier. For practical case of erosive wear during pneumatic conveying of particles, the typical impact velocity of the particles with the pipe wall is much smaller. Therefore, the validity of the model for smaller impact velocity was examined by comparing to the results of Arabnejad et al. [10], who study the erosion by sand particles impacting at velocity range of 9-28 m/s. In Arabnejad et al. [10] experiments the particles were semi-rounded sand particles $(\eta \approx 0.7$ equivalent to sharpness factor of 0.5 which was used by Arabnejad et al. [10]) of $150 \mu \mathrm{m}$ diameter. Fig. 13 presents the model prediction for erosion rate of aluminum 6061 alloy as a function of the impingement angle for different velocities. The hardness of aluminum 6061 was reported by Arabnejad et al. [10] to be $0.3 \mathrm{GPa}$ and its fracture toughness was estimated as $100 \mathrm{~kJ} / \mathrm{m}^{2}$. Fig. 13 shows good agreement between the developed model and the experimental data for the entire range of velocities, hence validating the model capability to perform in realistic wear scenarios during particle conveying. While these experiments measuring the maximum erosion angle cannot be entirely perceived due to lack of data points in the range of impingement angles smaller than $15^{\circ}$, the model prediction for maximum erosion angle of about $15^{0}$ seems to agree with the experimental data. This maximum erosion angle for aluminum 6063 was also reported by Desale et al. [18]. The model coefficients which received 
are $f=0.9, C_{D}=0.005, C_{C}=0.003$. Comparison of the received model coefficients from this case to the correlated function of section 4 for aluminum $6061\left(\mathrm{H} / \mathrm{R}=3 \mathrm{~mm}^{-1}\right)$ yields that the values of $C_{D}$ and $f$ are in very good agreement to the correlated functions.

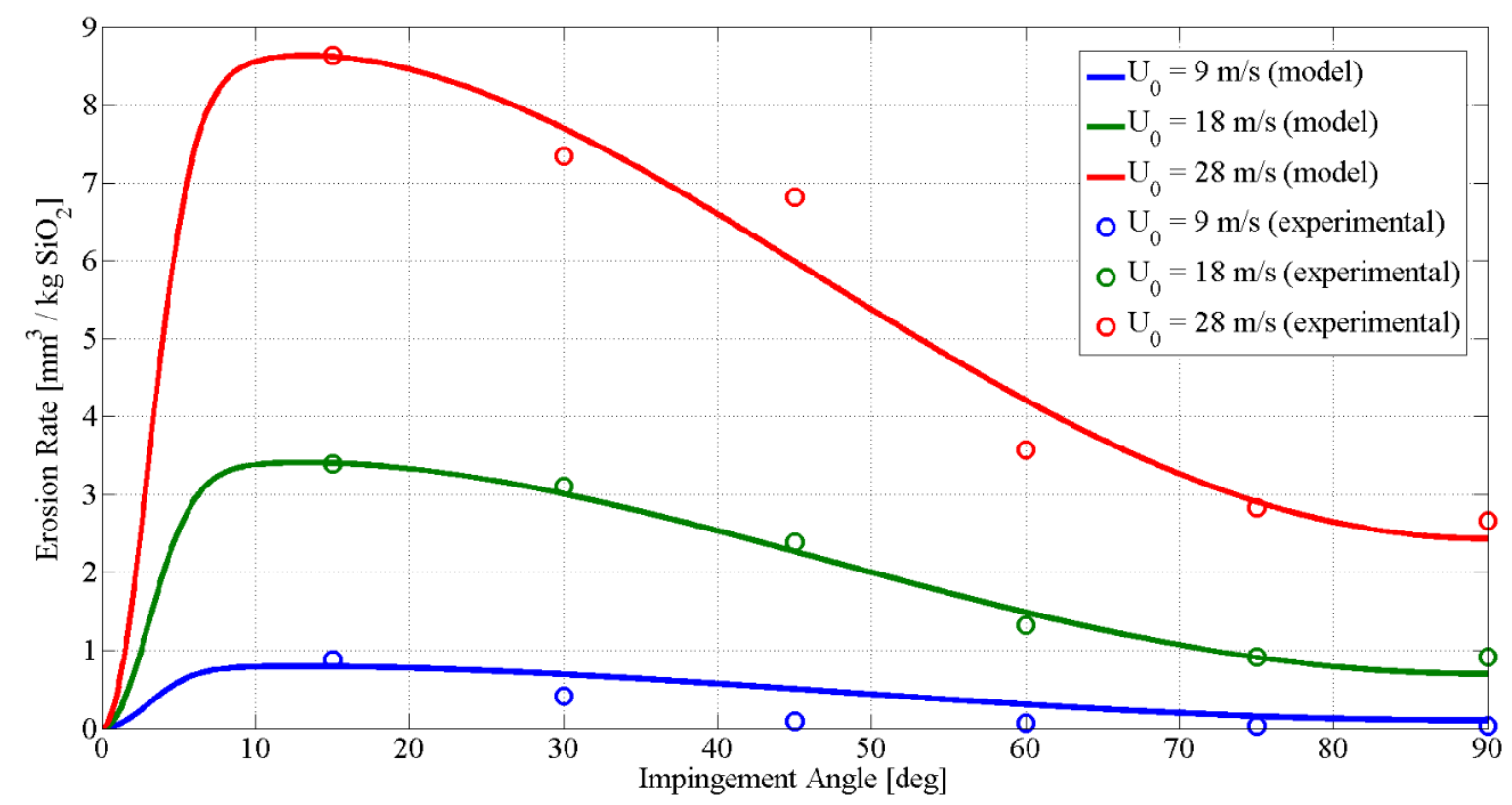

Fig. 13. Erosion rate of aluminum 6061 as a function of the impingement angle for different impact velocities. Model prediction vs. Arabnejad et al. [10] experimental results.

However, the value of $C_{C}$ is about 4 times smaller than the value received in Oka et al. [15],[16] experiments. This can be explained by the different sand types used in the experiments: semirounded shape in Arabnejadet al. [10] experiments and angular shape in Oka et al. [15],[16] experiments. Semi-rounded particles have larger effective diameter than angular particles which reduces the effectiveness of the ploughing mechanism. Further research should be done in order to quantify the effect of the particle shape on the erosion rate.

\section{Conclusions}

A semi-mechanistic, semi-empirical erosion model was developed. The newly developed model introduces the $f$ exponent which depends upon the ratio of the target material fracture toughness to its hardness and to the effective particle diameter, which is a measure for the particle sharpness. The $f$ exponent was shown to control the mechanism of material removal by horizontal forceploughing mechanism or fracture mechanism. This phenomenon was not predicted in the Huang et al. [9] model and the advantage of the new developed model was shown. The velocity exponent for this model lies in the range of $2 \div 3$, which correlate well with experimental findings. The transition between plastic deformation and fracture mechanism gives reasonable explanation to this phenomenon. The model also predicts exponential relation of $-0.5 \div-1$ between 'cutting' erosion and material hardness, which is in very good agreement with experimental data. 
Correlation between the developed erosion model coefficients to target material properties was examined using Oka's et al. [15],[16] erosion data. It was assumed that hardness alone is not sufficient in order to predict erosion rate and to explain different phenomenon, which occurs in erosion process, but the influence of fracture toughness have to be taken into account. The results received for the dependence of the model coefficients to the ratio $H / R$ showed good correlations that suitable for the physical interpretation of the coefficients. The $f$ exponent presented inverse logarithmic relation with $H / R$, which is supported by analysis of the work done by the particle and applying minimum energy criterion. The 'deformation' erosion mechanism is shown to be governed by the material ductility, as expected from mechanism of fatigue crack propagation. The material properties that control the extent of this mechanism (expressed by the magnitude of $C_{D}$ ) is also the ratio of hardness to fracture toughness, $H / R$. The cutting mechanism coefficient, $C_{C}$, showed no dependent on either target material property and was constant within a reasonable range.

The relations between target material mechanical properties to the model coefficients received in this research can be used for erosion prediction by angular $\mathrm{SiO}_{2}$ particles of various target materials, with large range of mechanical properties. Nevertheless, in order to extend the model to other types of abrasive particles, a further research should be done to quantify the effect of the effective diameter.

\section{References}

[1] Njobuenwu, D. O., \& Fairweather, M. (2012). Modelling of pipe bend erosion by dilute particle suspensions. Computers \& Chemical Engineering, 42, 235-247.

[2] Parsi, M., Najmi, K., Najafifard, F., Hassani, S., McLaury, B. S., \& Shirazi, S. A. (2014). A comprehensive review of solid particle erosion modeling for oil and gas wells and pipelines applications. Journal of Natural Gas Science and Engineering, 21, 850-873.

[3] Finnie, I. (1960). Erosion of surfaces by solid particles. Wear, 3(2), 87-103.

[4] Bitter, J. G. A. (1963). A study of erosion phenomena part I. wear, 6(1), 5-21.

[5] Bitter, J. G. A. (1963). A study of erosion phenomena: Part II. Wear, 6(3), 169-190.

[6] Hutchings, I. M. (1981). A model for the erosion of metals by spherical particles at normal incidence. Wear, 70(3), 269-281.

[7] Neilson, J. H., \& Gilchrist, A. (1968). Erosion by a stream of solid particles. Wear, 11(2), 111-122.

[8] Brach, R. M. (1988). Impact dynamics with applications to solid particle erosion. International journal of impact engineering, 7(1), 37-53.

[9] Huang, C., Chiovelli, S., Minev, P., Luo, J., \& Nandakumar, K. (2008). A comprehensive phenomenological model for erosion of materials in jet flow. Powder Technology, 187(3), 273-279.

[10] Arabnejad, H., Mansouri, A., Shirazi, S. A., \& McLaury, B. S. (2015). Development of mechanistic erosion equation for solid particles. Wear, 332, 1044-1050.

[11] Finnie, I. (1972). Some observations on the erosion of ductile metals. Wear, 19(1), 81-90.

[12] Levy, A. V. (1986). The platelet mechanism of erosion of ductile metals. Wear, 108(1), 1-21. 
[13] Hutchings, I. M. (1992). Tribology: friction and wear of engineering materials. Edward Arnold, London.

[14] Hornbogen, E. (1975). The role of fracture toughness in the wear of metals. Wear, 33(2), 251259.

[15] Oka, Y. I., Ohnogi, H., Hosokawa, T., \& Matsumura, M. (1997). The impact angle dependence of erosion damage caused by solid particle impact. Wear, 203, 573-579.

[16] Oka, Y. I., Okamura, K., \& Yoshida, T. (2005). Practical estimation of erosion damage caused by solid particle impact: Part 1: Effects of impact parameters on a predictive equation. Wear, 259(1), 95-101.

[17] Ahlert, K. R. (1994). Effects of particle impingement angle and surface wetting on solid particle erosion of AISI 1018 Steel (Doctoral dissertation, University of Tulsa).

[18] Desale, G. R., Gandhi, B. K., \& Jain, S. C. (2006). Effect of erodent properties on erosion wear of ductile type materials. Wear, 261(7), 914-921.

[19] Head, W. J., \& Harr, M. E. (1970). The development of a model to predict the erosion of materials by natural contaminants. Wear, 15(1), 1-46.

[20] Hutchings, I. M. (1977). Deformation of metal surfaces by the oblique impact of square plates. International Journal of Mechanical Sciences, 19(1), 45-52.

[21] Atkins, A. G., \& Liu, J. H. (2007). Toughness and the transition between cutting and rubbing in abrasive contacts. Wear, 262(1), 146-159.

[22] Chiu, W. C., Thouless, M. D., \& Endres, W. J. (1998). An analysis of chipping in brittle materials. International Journal of Fracture, 90(4), 287-298.

[23] Atkins, A. G. (2003). Modelling metal cutting using modern ductile fracture mechanics: quantitative explanations for some longstanding problems. International Journal of Mechanical Sciences, 45(2), 373-396.

[24] Sheldon, G. L., \& Kanhere, A. (1972). An investigation of impingement erosion using single particles. Wear, 21(1), 195-209.

[25] Meggiolaro, M. A., \& Castro, J. T. P. (2004). Statistical evaluation of strain-life fatigue crack initiation predictions. International Journal of Fatigue, 26(5), 463-476.

[26] Kobayashi, S., \& Thomsen, E. G. (1959). Some observations on the shearing process in metal cutting. ASME J. Ind, 251-261.

[27] Atkins, T. (2009). Toughness and processes of material removal. Wear, 267(11), 1764-1771.

[28] Salzbrenner, R. (1987). Fracture toughness behavior of ferritic ductile cast iron. Journal of materials science, 22(6), 2135-2147.

[29] Hornbogen, E. (1985). Fracture toughness and fatigue crack growth of grey cast irons. Journal of materials science, 20(11), 3897-3905.

[30] Ledbetter, H. M. (1982). Physical Properties Data Compilations Relevant to Energy Storage. National Bureau of Standards.

[31] Sundararajan, G., \& Shewmon, P. G. (1987). The oblique impact of a hard ball against ductile, semi-infinite target materials - experiment and analysis. International journal of impact engineering, 6(1), 3-22.

[32] Papini, M., \& Spelt, J. K. (2000). Impact of rigid angular particles with fully-plastic targets Part II: Parametric study of erosion phenomena. International Journal of Mechanical Sciences, 42(5), 1007-1025. 
[33] Sundararajan, G. (1990). The energy absorbed during the oblique impact of a hard ball against ductile target materials. International Journal of Impact Engineering, 9(3), 343-358. 